\title{
Electrical Tree Simulation Based on the Self-Organization Criticality
}

\author{
H. M. Hu, Y. Yang, W. Lu, G. P. Zhao \\ China Three Gorges University, Yi Chang, China \\ Email: yy46495027@163.com
}

Received April, 2013

\begin{abstract}
So far much effort has been made to understand the development of electrical treeing. For the simulation based study of electrical treeing, the most common method is to apply DBM stochastic model to simulate the growing of electrical treeing patterns. Previous simulation results showed that this stochastic model is capable of simulating the real electrical treeing patterns in a point-to-plane electrode system. However, this model only allows the tree channels to propagate on equipotential lines proportional to local electrical field. Therefore, it is necessary to develop a novel stochastic model to simulate the electrical patterns in order to get a good agreement with experimental results.
\end{abstract}

Keywords: Fractal; Electrical Tree simulation; Self-Organization Criticality

\section{Introduction}

Electrical treeing is a common pre-breakdown phenomenon for solid insulating materials when materials are subjected to high electrical stress. Electrical tree grows in a pattern resembling the branches of a tree. The branchlike structures are conductive channels formed through partial discharge activities. Experience from field and factory has shown that electrical treeing accounts for one of the main sources of long-term deterioration of insulating materials. Therefore, it is of great importance to study the electrical treeing phenomena in insulating materials for the improvement of reliability and safety of power system.

One of the major causes for polymer insulation degradation in a long time is electrical tree growth, the phenomenon of electrical tree growth contains discharge channels presented tubular random branching growth and partial discharge, which causes damage resulting in new formations of plasma channel, vice versa discharge structures determine the trajectory and the parameters of partial discharge, so electrical tree and partial discharge are the self consistent process of insulation degradation. Dynamic process of electrical tree growth and characteristics of partial discharge depend on the applied voltage's amplitude, frequency, electrode shape, physical properties of the medium, temperature and so on.

\section{The Traditional Method}

Niemeyer, Pietronero and Wiesmann Build a model when they study Flash discharge along surface smooth, short for NPW model [1]. In the model, electric field control indirectly discharge development but impact it through stochastic process, introducing discharge branch development probability in statistical sense, namely discharges take place at points where development probability is largest rather than electric field, which change the whole process from deterministic to stochastic. The relationship between the probability and electric field reflect that the overall discharge structure regulate the micro mechanism of discharge process, voltage drop along the discharge channel is less than the applied voltage, thus the formation of the channel is approximately close to potential structure, electric field distributes in symmetry of the circular and external boundary is quite a long way from discharge graphics.

The NPW model provide that discharge pattern grows stepwise from the cathode to the anode, electric potential $\phi$ of each grid unit is calculated by Laplace's equation with the boundary conditions as following[2]:

$$
\begin{cases}\phi=0 & \text { (center dot }) \\ \phi=1 & \text { (round edge) }\end{cases}
$$

In the grid square, the poor score of unit is calculated approximately in finite difference method througy following fomula:

$$
\phi_{x, y}=\frac{1}{4}\left(\phi_{x+1, y}+\phi_{x-1, y}+\phi_{x, y+1}+\phi_{x, y-1}\right)
$$

the probability $P$ is weight function to select candidate units according to electric potential, potential difference between candidate units and electrical tree points is asso- 
ciated with the development probability:

$$
p\left(i, k \rightarrow i^{\prime}, k^{\prime}\right)=\frac{\left(\phi_{i^{\prime}, k^{\prime}}\right)^{\eta}}{\sum\left(\phi_{i^{\prime}, k^{\prime}}\right)^{\eta}}
$$

The essence of the model is that the probability of electrical tree growth depend on local field determined by discharge pattern, the key part is to solve Laplace equation in each process step by step growth at a time, which reflects the impact of the new unit potential[5].

\section{Self-Organization Criticality}

The growth of electrical tree has the characteristics of self-organization critical phenomenon and self organization critical problem was put forward by Bake [3, 4] in the research of sand of cellular automata model. The state consisting of a square piece of sand is imagined that slope distribution decision. Slope of random point in the area is of increased to simulate the increasing number of grains of sand. This will lead to change around a few points of the slope when it is more than the threshold value of grains of sand can slide to a lower position. However, the redistribution of the slope is caused by the sand movement and further collapse of sand. As the grains of sand sliding, sand pile will tend to a dormant state at this time of infinite slope near threshold .Gradually increasing number of grains of sand will lead to different scale of sand pile collapse. But the system is still in the stationary state near collapse because size cannot be thought of system is in critical state. Main characteristic of self-organization critical system is developing to a critical state spontaneously under the influence of external factors. In this state there is no specific measurement, space or time and constant input produced the intermittent output as well as conforming to no specific proportion of the power law distribution.

From the perspective of self-organized criticality, along the electrical tree distribution channel of the charge is a spatial extension of dynamic system and presents a spatial extension of dynamic systems. Partial discharge is the output of the whole system as well as external factors as the applied voltage. It is necessary to consider about non-locality, nonlinear contact of space charge and restriction of electrical tree in charge movement and so on.

\section{The Simulation Principle}

For any kind of insulation degradation mechanism, the dielectric damage is caused by partial discharge the energy released and the local field strength. Therefore the degradation mechanism of rules need to consider the above two aspects. Discharge channel formation rules of the judge need to consider medium damage accumulation and random electrical tree growth. Probability distribution is used to describe the random growth, and then it based on the experimental data analysis of physical mechanism of insulation damage, it calculates the probability of discharge channel development and discharge energy, the relationship between electric field strength. When local damage energy or local field strength is lower than the critical value, the development of the probability is zero. And when the damage energy reaches critical value and the local field strength more than the critical value, the existing electrical points in the tree will form a new discharge channel. Many a branching pattern of one dimensional point is used to simulate electrical tree and point $d$ of the connection between the distances determines the minimum space simulation ratio. Discrete time constant $\Delta t$ is used to describe the transient process of electrical tree growth as well as time step $n$ corresponding to instantaneous time $t_{n}=n \cdot \Delta t$. The point $\mathrm{i}$ corresponds to the charge $q_{i}^{n}$ and specific energy $W_{i}^{n}$ and at each discrete time points in the electrode potential will change. Existing electrical tree structure or the increase of the electrode tip point is the simulation of electrical tree growth which depends on the electric field strength and the energy distribution of the damage. However, partial discharge will lead to field strength, failure energy, and charge distribution of change. The process time lasted until the electrical tree structure come into contact with the other electrode.

Every point of the electric potential is equal to all the charge voltage superposition, the i-th point on the potential of a discrete time step $\mathrm{n}$ can be expressed as following[6]:

$$
\begin{aligned}
\varphi^{n}{ }_{i}= & \phi_{j}^{n}+\sum_{j \neq i} \frac{q^{n}{ }_{j}}{4 \pi \varepsilon \varepsilon_{0}\left|\overrightarrow{\mathrm{r}}_{i}-\overrightarrow{\mathrm{r}}_{j}\right|} \\
& -\sum_{j} \frac{q^{n}{ }_{j}}{4 \pi \varepsilon \varepsilon_{0}\left|\overrightarrow{\mathrm{r}}_{i}-\overrightarrow{\mathrm{r}}_{j}{ }^{*}\right|}
\end{aligned}
$$

$\varepsilon_{o}$ is the vacuum dielectric constant, $\varepsilon$ is the relative dielectric constant, $\overrightarrow{r_{i}}, \overrightarrow{r_{j}}$ is the position vector, $\phi_{i}^{n}$ is a part of point charge electric potential $q_{i}^{n}$, relationship lies on the size of the electric field distributed around electrical tree channel, The minimum measure in space adopts the size of space charge area, $\phi_{i}^{n}$ is expressed in Spherical charge formula:

$$
\phi_{i}^{n}=\frac{q_{i}^{n}}{2 \pi \varepsilon \varepsilon_{0} \mathrm{~d}}
$$

Specific damage energy $W_{\mathrm{i}}^{\mathrm{n}}$ of any i-th point at existing electrical trees reaches a critical value $W_{\mathrm{c}}$, there is a new point is attached to the structure. Several additional locations are randomly situated around i-th point, the gaps between them and i-th point are not less than bond length $\mathrm{d}$, and the selection of lately additional position among certain number of impossible locations is in 
accordance with development probability $\mathrm{P}$ that is expressed as following [7]:

$$
P=Z^{-1}(\Delta \varphi)^{2} \theta\left(\Delta \varphi-E_{\mathrm{c}} \mathrm{d}\right) \theta\left(\mathrm{W}_{i}^{n}-W_{c}\right)
$$

$Z=\sum(\Delta \varphi)^{2}$ is normalization factor, summation is made over all possible attachment positions providing $\Delta \varphi>E_{c} d, \theta(x)$ is step function, $\theta(x)=0$ if $x \leq 0$, $\theta(x)=1$ if $x>0$. The first Growing point attach solely to electrode at the beginning of the electrical tree growth.

Assume that there is only a partial discharge at each time step, so the time interval should be small enough so as to distinguish the different partial discharge. Partial discharge is the process in which all kinds of factors influence each other, involving charge, self-consistent calculation of electrical field and the change of boundary conditions [9], in each interactive time step $\mathrm{k}$, growing point is characterized with charge $q_{i}^{k}(k)$ and specific damage energy $W^{k}{ }_{i}(k)$. Segment between $\mathrm{i}$ and $\mathrm{j}$ point is characterized with status value $S_{i, j}^{n}(k)$, there are two distinct states: conducting $S_{i, j}^{n}(k)=1$ and non-conducting $S_{i, j}^{n}(k)=0$, conducting state correspond to the discharge. The electric potentials of point charge are calculated according to equation (1), at the beginning of the discharge the point states are determined by the previous time step, namly $q_{i}^{n}(0)=q_{i}^{n-1}, W_{i}^{n}(0)=W_{i}^{n-1}$, all bonds are in the non-conducting state $S_{i, j}{ }^{n}(0)=0$.

The bond in the non-conducting state transits into the conducting state if the absolute value of the potential difference $\Delta \varphi_{i, j}^{n}(k)$ exceeds the inception voltage $\Delta \varphi_{\text {ign }}[8]:$

$$
\left|\varphi^{n}{ }_{i}(k)-\varphi^{n}{ }_{j}(k)\right|>E_{\text {ign }} \cdot d
$$

The bond in the conducting state transits into the nonconducting state if the absolute value of the potential difference $\Delta \varphi_{i, j}^{n}(k)$ falls below the residual voltage $\Delta \varphi_{\text {res }}$ :

$$
\left|\varphi^{n}{ }_{i}(k)-\varphi^{n}{ }_{j}(k)\right|<E_{\text {res }} \cdot d
$$

The bond state at time step $\mathrm{k}+1$ is expressed by the step function $\theta(x)$ as following:

$$
\begin{aligned}
S_{i, j}^{n}(k+1)= & S_{i, j}^{n}(k) \cdot \theta\left(\left|\Delta \varphi_{i, j}^{n}(k)\right|-\Delta \varphi_{\text {res }}\right) \\
& +\left(1-S_{i, j}^{n}(k)\right) \cdot \theta\left(\left|\Delta \varphi_{i, j}^{n}(k)\right|-\Delta \varphi_{i g n}\right)
\end{aligned}
$$

Charge transfer takes place along the bonds in the conducting state; charge value $\Delta q_{i, j}^{n}(k+1)$ is proportional to the potential difference among points:

$$
\Delta q_{i, j}^{n}(k+1)=\lambda \cdot S^{n}{ }_{i, j}(n+1) \cdot \Delta \varphi_{i, j}^{n}(k)
$$

$\lambda$ is a numerical parameter that determines speed and accuracy of the process, the new charge distribution is given by:

$$
q_{i}^{n}(k+1)=q_{i}^{n}(k)-\sum_{j} \Delta q_{i, j}^{n}(k+1)
$$

The charge movement will release damage energy to form new plasma channel the specific damage energy $W_{i}^{n}(k+1)$ at time step $\mathrm{k}+1$ can be expressed as:

$$
\begin{aligned}
& W_{i}^{n}(k+1) \\
= & W_{i}^{n}(k)+\Delta q_{i, j}^{n}(k+1) \cdot \Delta \varphi_{i, j}^{n}(k) / d
\end{aligned}
$$

Partial discharge path is composed of contact state between all of the above points, discharge process will be terminated when all bonds are in non-conducting.

\section{Conclusions}

Traditional methods calculate the electric field distribution in means of Solving Laplace equation or Poisson's equation in Lattice squares, but the disadvantage of this approach is the electrical tree growth is limited on the grid of line and diagonal direction, electrical tree channel diameter is equal to the size of grid line, the simulation only probably reflects the rough trend of discharge. In this paper a novel stochastic model based on analyses of self-organized criticality of electrical treeing was applied to simulate the formation of electrical tree. Fractal dimension and multi-fractal spectrum of simulated electrical treeing patterns were calculated by using boxing-counting method. The relationship between the fractal characteristics of the simulated electrical treeing patterns and those of real electrical treeing patterns were finally defined.

\section{REFERENCES}

[1] L. Niemeyer, L. Pietronero and H. J. Wiesmann, "Fractal Dimension of Dielectric Breakdown", Physical Review Lett., Vol. 52, No. 12, 1984, pp. 1033-1036. doi:0.1103/PhysRevLett.52.1033

[2] S. P. Frankel (1950). Convergence rates of interactive treatments of partial differential equations. Mathematical Tables and Other Aids to Computation 4, pp. 65-75. doi:10.2307/2002770

[3] P. Bak, C. Tang and K. Wiesenfeld 1987 Phys. Rev. Lett. 59.381-384

[4] P. Bak, C. Tang and K. Wiesenfeld, "Self-organized Criticality,” Physical Review A, Vol. 38, 1988, pp. 364-374.doi:10.1103/PhysRevA.38.364

[5] J. M. Cooper and G. C. Stevens, "The Influence of Physical Properties on Electrical Treeing in a Cross-linked Synthetic Resin,” Journal of Phys. D: Appl. Phys., Vol. 23, 1990, pp. 1528-1535.

[6] H. J. Wiesmann and H. R. Zeller, “A Fractal Model of Dielectric Breakdown and Prebreakdown in Solid Dielectrics,” Journal of Applied Physics, Vol. 60, No. 5, 1986, pp. 1770-1773. doi:10.1063/1.337219

[7] L. Kebbabi and A. Beroual, "Fractal Analysis of Creeping Discharge Patterns Propagating at Solid/liquid Interfaces: Influence of the Nature and Geometry of Solid Insula- 
tors,” Journal of the Physics D: Applied Physics, Vol. 39, 2006, pp. 177-183. doi:10.1088/0022-3727/39/1/026

[8] P. Wlezek, A. Odgaard and M. Sernetz, "Fractal 3D Analysis of Blood Vessels and Bones,” Fractal Geometry and Computer Graphics, Springer-Verlag, Berlin, pp.
240-248, 1992.

[9] K. Kudo, "Fractal Analysis of Electrical Trees," IEEE Transactions on Dielectrics and Electrical Insulation, Vol. 5 No. 5, October 1998, PP. 713-727.

doi:10.1109/94.729694 